\title{
Accidente cerebrovascular isquémico asociado a COVID-19: primer reporte de casos en Perú.
}

\author{
SARS-CoV-2 associated ischemic stroke: first case report in Peru. \\ Evelyn Mariños 1, a , Elliot Barreto-Acevedo", a, Poul Espino, a

\section{RESUMEN}

La enfermedad causada por el coronavirus 2 asociado al síndrome respiratorio agudo grave (SARS-CoV-2) se conoce como Coronavirus Disease-2019 (COVID-19). Su afectación es principalmente respiratoria, pero se han descrito diversas manifestaciones neurológicas. El neurotropismo y potencial rol neuropatógeno del SARS-CoV-2, se plantea en base a modelos en animales y casos de afección neurológica descritos en otros coronavirus, así como por reportes recientes de síndromes neurológicos específicos relacionados a COVID-19, incluido el accidente cerebrovascular (ACV).

La sociedad española de neurología(SEN) describió a los ACV isquémicos como la segunda manifestación neurológica más frecuente en pacientes con COVID-19. Se reconoce que el estado de hiperinflamación e hipercoagulabilidad sanguínea desencadenada por el COVID-19 incrementaría el riesgo de eventos protrombóticos.

Reportamos 3 casos de pacientes peruanos que presentaron ACV isquémico asociado a COVID-19: describimos el cuadro clínico, exámenes paraclínicos y revisamos la literatura mundial.

PALABRAS CLAVE: Accidente cerebrovascular, Infarto cerebral, COVID-19, SARS-CoV-2.

\section{SUMMARY}

The disease caused by Severe Acute Respiratory Syndrome Coronavirus 2 (SARS-CoV-2) is known as Coronavirus Disease 2019 (COVID-19). Its involvement is mainly respiratory, but various neurological manifestations have been described. The neurotropism and potential neuropathotogenic role of SARS-CoV-2 is based on animal models and cases of neurological condition described in other coronaviruses, as well as recent reports of specific neurological syndromes related to COVID-19, including ischemic stroke. The Spanish Society of Neurology described ischemic stroke as the second most frequent neurological manifestation in patients with COVID-19. Likewise, the state of hyperinflammation and blood hypercoagulability triggered by COVID-19 would increase the risk of prothrombotic events. We report 3 cases of peruvian patients who presented ischemic stroke associated with COVID-19: we describe the clinical manifestations, paraclinical examinations and review the world literature.

KEYWORDS: Cerebrovascular accident, Ischemic stroke, COVID-19, SARS-CoV-2

Departamento de Neurología, Hospital Nacional Edgardo Rebagliati Martins, EsSalud. Lima, Perú.

a Médico Asistente Neurólogo 


\section{INTRODUCCIÓN}

La enfermedad causada por el coronavirus 2 asociado al síndrome respiratorio agudo grave (SARSCoV-2) se conoce como Coronavirus Disease 2019 (COVID-19); esta enfermedad es nueva en el mundo, y sus orígenes se remontan a diciembre del 2019 cuando en Wuhan, provincia de Hubei en China, se reportó uno serie de casos con neumonía grave cuyo agente etiológico recién se descubrió en enero del 2020. La enfermedad principalmente tiene afectación respiratoria, pero ya en ese primer reporte se hace referencia a manifestaciones neurológicas, como cefalea $(1,2)$.

El neurotropismo y el potencial papel neuropatógeno del SARS-CoV-2 se plantea en base a modelos en animales y casos de afección neurológica por coronavirus previos (SARS-CoV, MERS CoV, HCoV-OC43); además por reportes recientes de casos con síndromes neurológicos específicos como Guillain Barre y encefalitis asociados a COVID-19 (3-7).

El SARS-CoV-2 a través de su proteína S se fija al receptor de la enzima convertidora de angiotensina 2 (ECA-2) para invadir las células y replicarse. El receptor de la ECA-2 también se expresaría en el sistema nervioso central (neuronas, glía y endotelio) según estudios en animales. Hoy en día, se desconoce si los accidentes cerebro-vasculares (ACV) relacionados al SARS-CoV-2 se deba a un efecto directo del virus o indirecto, relacionado con el efecto pro-trombótico de la respuesta inflamatoria, tal como se ha descrito en otras infecciones por virus y bacterias (8-9).

En un estudio de 214 pacientes con COVID-19 en China, el 36,4\% (78 pacientes) presentaron síntomas neurológicos, además en este estudio se describen 5 pacientes con ACV isquémicos (10). Posteriormente se publicó una serie de casos de 13 pacientes con COVID-19 y ACV, de los cuales 11 fueron isquémicos. Asimismo, la Sociedad Española de Neurología (SEN) en una serie de 92 pacientes con COVID-19 describió el ACV isquémico cómo la segunda manifestación neurológica más frecuente con el 22,8\%. Estas publicaciones ponen en evidencia que la concurrencia de ACV isquémico y COVID-19 no son tan infrecuentes (9-11).

En nuestro país, se confirmó la primera persona infectada por SARS-CoV-2 el 6 de marzo de 2020. A la fecha hay 2 estudios descriptivos sobre las características clínicas de los pacientes con COVID-19 en 2 centros de Lima, dónde no reportan ACV (12-14). A continuación, describiremos 3 casos de accidente cerebrovascular isquémico asociado a COVID-19 atendidos en el hospital Nacional Edgardo Rebagliati Martins-EsSalud.

\section{Caso 1}

Varón de 74 años, diestro, autosuficiente, hipertenso y con dislipidemia, en tratamiento con losartán $100 \mathrm{mg} /$ día y atorvastatina $20 \mathrm{mg}$ /día. Se encontraba cumpliendo cuarentena en casa. Ingresó a la emergencia del hospital con tiempo de enfermedad de 4 horas, con cuadro clínico de inicio brusco caracterizado por trastorno del nivel de consciencia y alteración del lenguaje. En el examen neurológico se encontró somnoliento, con desviación de la mirada conjugada a la izquierda, afasia de predominio motora, hemiplejía derecha y Babinski derecho; el sistema cardiovascular y respiratorio estuvieron normales, los signos vitales fueron normales, salvo febrícula. La tomografía computarizada (TC) cerebral (figura 1), a su ingreso reveló signos tempranos de evento isquémico en el territorio de la arteria cerebral media (ACM) izquierda, y la TC a las 24 horas evidenció infarto cerebral en el territorio de la división superior de la ACM izquierda. El paciente recibió antiagregación con ácido acetil salicílico $100 \mathrm{mg}$ /día, además atorvastatina a 40mg/día. Al siguiente día de la hospitalización cursó febril, luego de 3 días se añadió polipnea y disnea, requiriendo apoyo de oxigeno suplementario por cánula binasal. En los resultados analíticos destacó hemograma con leucocitosis (15 320/ul) y linfopenia relativa $(7 \%)$, dímero $\mathrm{D}$ alto $(7,16 \mathrm{ug} / \mathrm{ml})$ y $\mathrm{PCR}$ elevada $(9.40 \mathrm{mg} / \mathrm{dl})$ (tabla 1$)$. La tomografía de tórax (figura 1) mostró opacidades en vidrio esmerilado, de distribución predominantemente periférica subpleral en especial en segmentos posteriores de ambos campos pulmonares. Se realizó la prueba serológica por inmunocromatografía para SARS-CoV-2 IgG/IgM, resultando reactiva para ambas inmunoglobulinas, además la RT-PCR para SARS-CoV-2 en muestra de hisopado nasofaríngeo fue positiva. El paciente recibió tratamiento de soporte con oxigenoterapia, además por sospecha de sobreinfección con neumonía bacteriana intrahospitalaria (por leucocitosis con neutrofilia en el hemograma y fiebre persistente) se añadió meropenem. Hubo mejoría clínica respiratoria luego de 10 días, quedando afebril y logrando ventilar a aire ambiental. Neurológicamente persistió con afasia y hubo recuperación parcial del déficit motor, no se realizaron mayores estudios cardiovasculares, excepto electrocardiograma el cual fue normal. A la fecha 


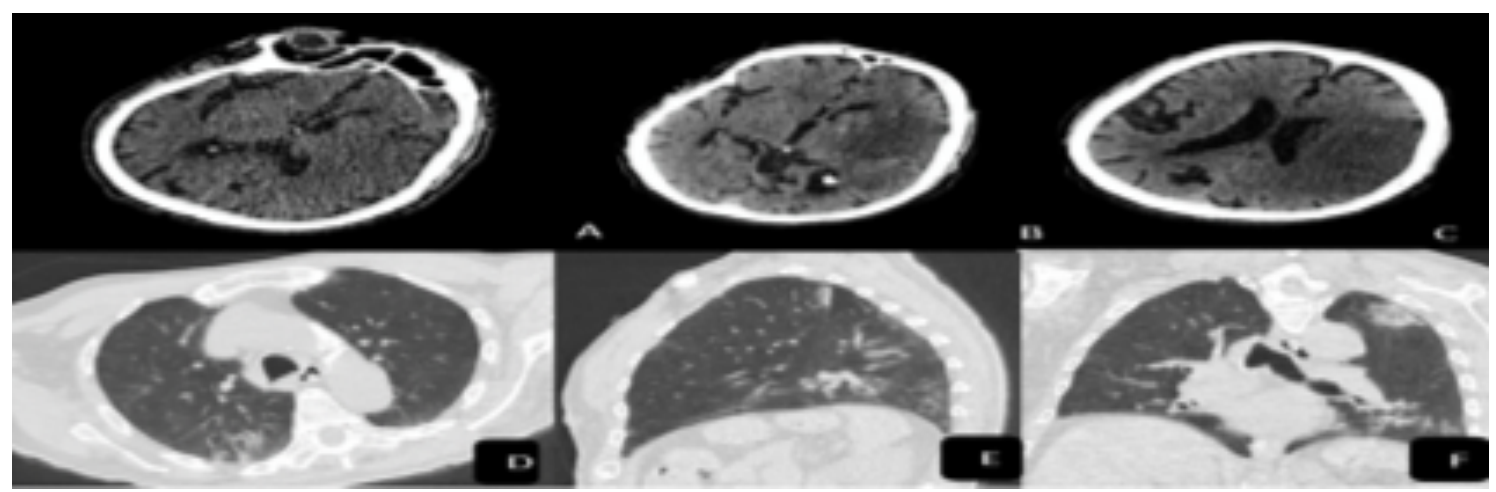

Figura 1.TC cerebral sin contraste con signos tempranos de infarto cerebral (A). Control de TC de 24 horas, con infarto en territorio de la ACM izquierda (B y C). TC de tórax sin contraste con opacidades en vidrio esmerilado periférico subpleural, posterior bilateral (D, E); asociado a zona focal de patrón empedrado (F).

de este reporte el paciente permanecía hospitalizado completando tratamiento antibiótico.

\section{Caso 2}

Mujer de 91 años, con fibrilación auricular sin anticoagulación, hipotiroidismo y úlcera duodenal no activa; en tratamiento con bisoprolol, digoxina, levotiroxina y omeprazol. Su estado basal era dependiente parcial para actividades de la vida diaria y describen deterioro cognitivo. Se encontraba cumpliendo cuarentena en casa. Inició síntomas 7 días previos al ingreso hospitalario, presentando diarrea no disentérica asociado a hiporexia, 3 días antes del ingreso notan debilidad del miembro superior izquierdo. A su ingreso por emergencia los signos vitales eran normales. Al examen neurológico se encontró somnolienta, desorientada en tiempo, espacio y persona, con hemiparesia izquierda no proporcional $4 / 5$, hemihipoestesia izquierda, Babinski izquierdo. Los ruidos cardiacos fueron arrítmicos, a la auscultación pulmonar se encontró crépitos bibasales.
Exámenes auxiliares: el electrocardiograma (ECG) evidenció fibrilación auricular, TC cerebral (figura 2), dónde se evidenció infarto cerebral en territorio de ACM derecha, TC de tórax (figura2) que mostró focos con densidad en vidrio esmerilado de distribución periférica bilateral, a predominio basal. La prueba serológica por inmunocromatografía para SARS-CoV-2 IgG/IgM, resultó reactiva para ambas inmunoglobulinas. Se complementó el estudio con analítica sanguínea resaltando linfopenia (763/ul), DHL elevada (418 U/L), dímero D elevado (10.6 ug/ml), PCR alto $(27.9 \mathrm{mg} / \mathrm{dl})$ (Tabla 1). Además, el RT-PCR para SARS-CoV-2 en muestra de hisopado nasofaríngeo fue positivo. La paciente recibió tratamiento de soporte con oxigenoterapia, asociado a hidroxicloroquina y aztiromicina, además se asoció ceftriaxona por sospecha de sobreinfección con neumonía bacteriana de la comunidad (por leucocitosis con neutrofilia en el hemograma y PCR elevada). A la fecha de este reporte la paciente permanece hospitalizada completando tratamiento antibiótico.

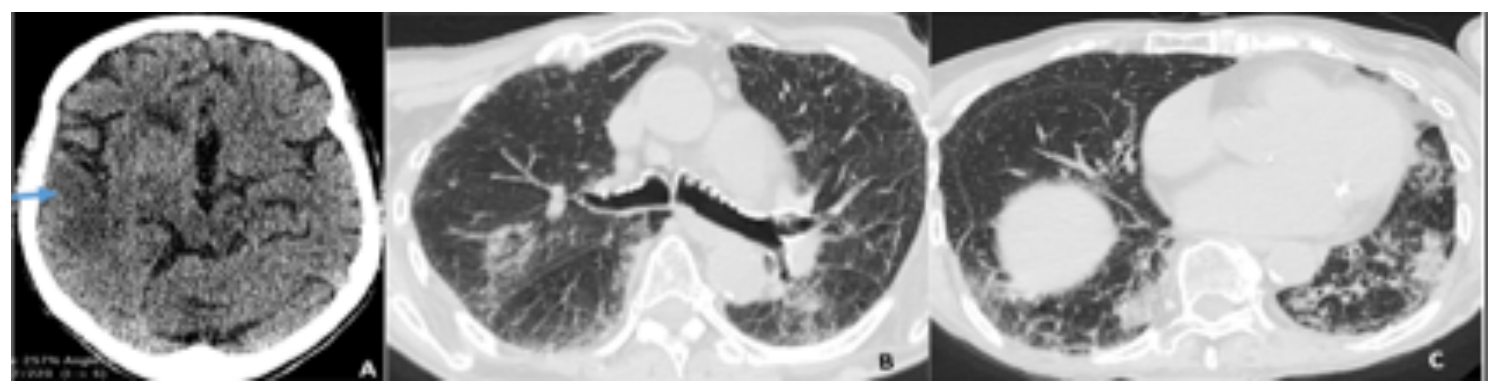

Figura 2. (A)TC de cerebro sin contraste, muestra infarto en región de ACM derecha. (B y C) TC de tórax muestra focos con densidad en vidrio esmerilado de distribución periférica bilateral a predominio basal, bronquiectasias en el lóbulo inferior izquierdo, asimismo zonas con engrosamiento del intersticio interlobulillar. 
Mariños E, et al.

Tabla 1. Características de los pacientes con Ataque Cerebral isquémico y COVID -19

\begin{tabular}{|c|c|c|c|}
\hline & CASO 1 & CASO 2 & CASO 3 \\
\hline Edad (años) & 74 & 91 & 61 \\
\hline Género & Masculino & Femenino & Femenino \\
\hline Comorbilidad & $\begin{array}{c}\text { HTA } \\
\text { Dislipidemia }\end{array}$ & $\begin{array}{l}\text { Fibrilación auricular } \\
\text { Hipotiroidismo } \\
\text { Úlcera duodenal no } \\
\text { active. }\end{array}$ & HTA \\
\hline $\begin{array}{l}\text { Clasificación TOAST del ACV } \\
\text { isquémico }\end{array}$ & Indeterminado & Indeterminado & Indeterminado \\
\hline 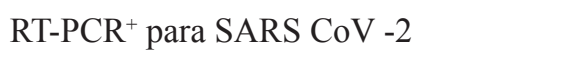 & Positivo & Positivo & Negativo \\
\hline Prueba serológica* para SARS CoV-2 & $\begin{array}{l}\text { IgM reactiva } \\
\text { IgG reactiva }\end{array}$ & $\begin{array}{l}\text { IgM reactiva } \\
\text { IgG reactiva }\end{array}$ & $\begin{array}{l}\text { IgM no reactiva } \\
\text { IgG reactiva }\end{array}$ \\
\hline Gravedad de COVID-19 & Severo & Severo & Crítico \\
\hline Inicio de síntomas de COVID 19 & Mismo día del ACV & 7 días previos al $\mathrm{ACV}$ & Mismo día del ACV \\
\hline Leucocitos en sangre (recuento/ul) & 15320 & 14690 & 17760 \\
\hline Linfocitos en sangre (recuento/ul) & 1190 & 763 & 830 \\
\hline $\operatorname{PCR}(\mathrm{mg} / \mathrm{dl})$ & 9.4 & 27.9 & 23.5 \\
\hline $\begin{array}{l}\text { Dímero D (ug/ml) } \\
\text { VR: }<0.54 \mathrm{ug} / \mathrm{ml}\end{array}$ & 7.6 & 10.6 & 34.6 \\
\hline DHL (U/L) & NR & 418 & 453 \\
\hline $\begin{array}{l}\text { TGO/TGP }(\mathrm{U} / \mathrm{L}) \\
\mathrm{VR}:<34 \mathrm{U} / \mathrm{L} /<49 \mathrm{U} / \mathrm{L}\end{array}$ & NR & $29 / 32$ & $34 / 32$ \\
\hline $\begin{array}{l}\text { Urea }(\mathrm{mg} / \mathrm{dl}) \\
\text { VR:22-55mg/dl }\end{array}$ & 42 & 118 & 64.7 \\
\hline $\begin{array}{l}\text { Creatinina }(\mathrm{mg} / \mathrm{dl}) \\
\text { VR:0.50- } 0.80 \mathrm{mg} / \mathrm{dl}\end{array}$ & 0.89 & 0.56 & 0.80 \\
\hline $\begin{array}{l}\text { CPK (U/L) } \\
\text { VR:34-145U/L }\end{array}$ & NR & 62 & 146 \\
\hline EKG & Ritmo sinusal & Fibrilación auricular & Ritmo sinusal \\
\hline Tratamiento Recibido & $\begin{array}{c}\text { Oxigenoterapia } \\
\text { Meropenem } \\
\text { AAS } \\
\text { Estatinas }\end{array}$ & $\begin{array}{c}\text { Oxigenoterapia } \\
\text { Azitromicina } \\
\text { Ceftriaxona } \\
\text { Hidroxicloroquina. }\end{array}$ & $\begin{array}{l}\text { Ventilación mecánica } \\
\text { Azitromicina } \\
\text { Ceftriaxona Manitol }\end{array}$ \\
\hline Resultado clínico & Aún hospitalizado & Aún hospitalizado & Fallecido \\
\hline
\end{tabular}

Abreviaciones: COVID-19: Enfermedad por coronavirus 2019, ACV: Ataque cerebrovascular, HTA: Hipertensión arterial, PCR: Proteína C reactiva; DHL: deshidrogenasa láctica; TGO: transaminasa glutámico oxalacética; TGP: Transaminasa glutámico piruvato; NR: no se realizó. * El método empleado para la prueba serológica fue la inmunocromatografía. RT-PCR+: Reacción en cadena de polimerasa en tiempo real de hisopado nasofaringeo. VR Valor referencial.

\section{Caso 3}

Mujer de 61 años, hipertensa sin tratamiento, que laboraba como vendedora en un mercado. Ingresó al hospital con tiempo de enfermedad de 24 horas, con cuadro clínico de inicio brusco caracterizado por debilidad en hemicuerpo derecho y trastorno del lenguaje. No se reportó clínica respiratoria. A su ingreso se encontró somnolienta, con afasia global, desviación de la mirada conjugada a la izquierda, hemiplejía derecha, hemihipoestesia derecha y Babisnki derecho. Se encontraba hipertensa $(147 / 67 \mathrm{mmHg})$, polipneica (24 respiraciones/minuto), y con saturación de oxígeno 93\%; además la auscultación pulmonar evidenció subcrépitos bibasales, la auscultación cardiaca fue normal. La TC cerebral (figura 3) de ingreso mostró el 


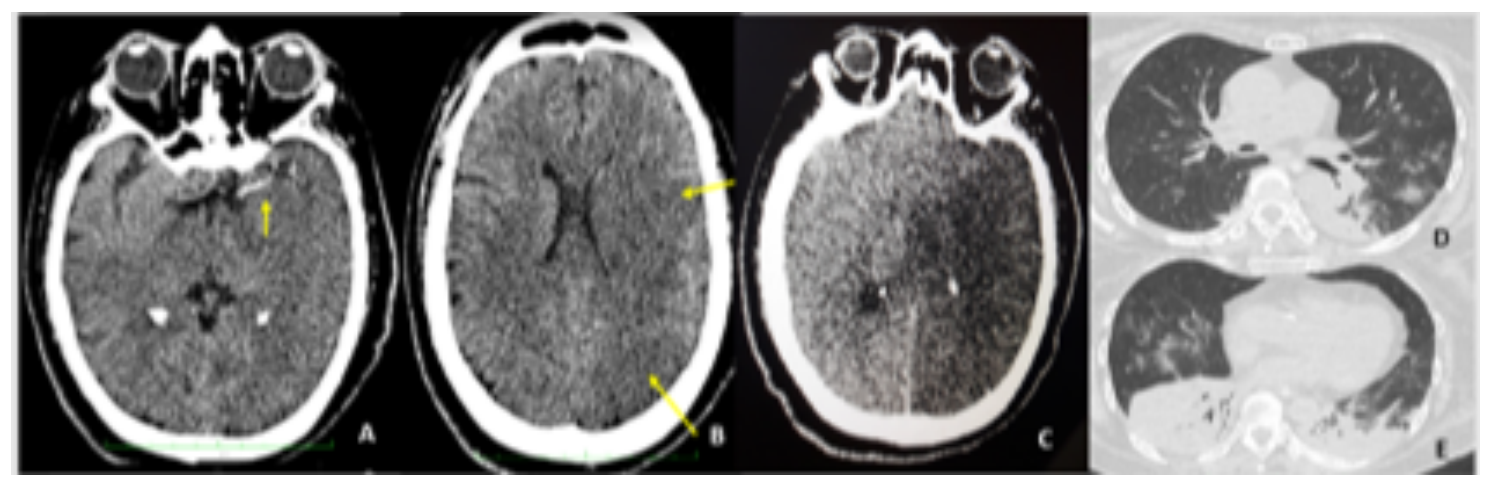

Figura 3. (A) TC cerebral sin contraste muestra hiperdensidad de la ACM izquierda. (B) hipodensidad extensa de la corteza del lóbulo fronto parieto tempo insular izquierdo. (C) Control a las 48 horas hipodensidad extensa y herniación subfalcina. (D y E) TC de tórax focos de vidrio esmerilado de distribución periférica bilateral y áreas de consolidación.

signo de la ACM izquierda hiperdensa y a las 48 horas infarto extenso del territorio ACM izquierda segmento M1. La TC de tórax (figura 3), evidenció algunas opacidades en vidrio esmerilado bilateral, además zonas de consolidación. La prueba serológica por inmunocromatografía para SARS-CoV-2 IgG/IgM, resultó reactiva para IgG y la RT-PCR para SARS$\mathrm{CoV}-2$ en muestra de hisopado nasofaríngeo fue negativa. La paciente recibió tratamiento de soporte, manitol, azitromicina, ceftriaxona; su evolución fue desfavorable, progresando a insuficiencia respiratoria requiriendo ventilación mecánica y shock séptico con necesidad de vasopresores. A pesar de todo, falleció al 4 día del ingreso, siendo cremada.

\section{DISCUSIÓN}

Los accidentes cerebrovasculares isquémicos se han relacionado a infecciones por diferentes virus, destacando los descritos en la enfermedad severa por SARS-CoV, ya que presenta una similitud en su secuencia genómica del $82 \%$ con el SARS-CoV-2 (15).

En la serie de casos de COVID-19 con manifestaciones neurológicas de la SEN, se reportó que 20 de 92 pacientes, tuvieron ACV isquémico, aunque no describieron a detalle las características de dichos pacientes (9). La serie de casos de ACV isquémico y COVID-19 con mayor número de pacientes, es la publicada por Yanan Li y colaboradores; los autores realizaron un estudio retrospectivo en un hospital de Wuhan; de 221 pacientes con COVID-19, 13 pacientes $(5,9 \%)$ tuvieron $\mathrm{ACV}$, de los cuales 11 fueron isquémicos. Estos pacientes fueron en su mayoría adultos mayores y con mayor prevalencia de factores de riesgo vascular (hipertensión arterial, diabetes o tabaquismo) comparados con los pacientes sin ACV. Además, dichos pacientes desarrollaron COVID-19 severo. A la fecha de publicación del artículo, 5 pacientes $(38,5 \%)$ habían fallecido y el resto permanecía hospitalizado (11).

Nuestro centro hospitalario desde el 11 de marzo hasta el 15 de abril presentó 153 pacientes hospitalizados con COVID-19 diagnosticados por RTPCR de muestra de hisopados nasofaríngeos. De estos, 3 pacientes $(1,9 \%)$ presentaron $\mathrm{ACV}$ isquémico.

Nuestros pacientes al igual que en el estudio de Li fueron adultos mayores, y tenían otros factores de riesgo vascular (hipertensión arterial: caso 1 y 3; dislipidemia: caso 1; fibrilación auricular caso 2). La gravedad del COVID-19 en estos pacientes se clasificó siguiendo las recomendaciones de la OMS y grandes series de casos publicadas $(16,17)$ resultando en: enfermedad severa: Caso 1 y 2 (con insuficiencia respiratoria) y, enfermedad crítica: caso 3 (necesidad de ventilación mecánica y shock séptico).

En los pacientes con COVID-19 severo y crítico se reportan más casos de $\mathrm{ACV}$. Un análisis agrupado de 4 estudios, mostró un incremento del riesgo de desarrollar enfermedad severa por SARS-CoV-2 en pacientes con ataques cerebrovasculares (18). En la serie de casos de Li, 10 de los 11 pacientes que desarrollaron ACV isquémico tuvieron COVID-19 severo. En una unidad de cuidados intensivos (UCI) de un hospital de Francia, de 58 pacientes admitidos, 3 pacientes $(5,1 \%)$ desarrollaron infartos cerebrales agudos por resonancia magnética, a pesar que durante su examen clínico no encontraron focalidad 
neurológica (19). Así mismo, en una UCI de un hospital de Holanda, de 25 pacientes con COVID-19 y complicaciones trombóticas, 3 pacientes $(12 \%)$ tuvieron ACV isquémico (20).

En diferentes estudios de pacientes de origen chino con COVID-19 se ha descrito como marcadores de mal pronóstico la presencia de linfopenia, incremento de DHL, elevación del dímero D y PCR. Así mismo se reconoce cada vez más con mayor frecuencia la inflamación como un contribuyente clave en la fisiopatología de las enfermedades cerebrovasculares (21-23). En nuestros pacientes estos marcadores de mal pronóstico estuvieron presentes, sobre todo en el caso 3, la cual falleció.

La etiología de los ACV isquémicos en los casos descritos, ha quedado como indeterrminado (Clasificación TOAST), pues debido al contexto de la pandemia, los estudios complementarios como ecocardiograma, holter, doppler carotideo entre otros están suspendidos, salvo extrema necesidad.

Se ha planteado que los eventos trombóticos aparecen en la tercera fase de la enfermedad por COVID-19, la cual ocurre a partir del día 10 o 14 (fase de hiperinflamación). En la serie descrita por Li el tiempo promedio entre los primeros síntomas de COVID-19 y la aparición del evento vascular fue 10 días. En nuestros pacientes, salvo el caso 2 (que tuvo síntomas digestivos 1 semana previo al evento vascular); en el caso 1 y 3, clínicamente las manifestaciones de infección se describieron el mismo día del evento vascular (caso 1 febrícula y caso 3 auscultación pulmonar anormal), sugiriendo que la enfermedad por SARS-CoV-2 y el evento vascular coincidieron. Sin embargo, hay 2 observaciones que refutan esto; la primera es que a su ingreso ya habían lesiones pulmonares en la TC de tórax en el caso 3 (es decir al menos estaría en fase pulmonar de la enfermedad que suele ocurrir a partir del día 7); y por otro lado las pruebas serológicas resultaron en IgM e IgG reactivas en los casos 1 y 2 , y solo $\operatorname{IgG}$ reactiva en el caso 3, este último además con RT-PCR de muestra de hisopado nasofaríngeo negativa. Se ha descrito la positividad de IgG a partir del día 14 de la enfermedad, además la RT-PCR se va negativizando luego de la primera semana, con sensibilidad de menos del 70\% luego de la segunda semana de enfermedad. Por lo anteriormente expuesto pensamos que el evento vascular ocurrió en fase avanzada de la enfermedad en los 3 casos con infección por SARS-CoV-2, dónde los problemas protrombóticos son mayores $(24,25)$.
En conclusión, nuestro estudio no permite esclarecer una relación causal entre el accidente cerebrovascular isquémico y COVID-19, pero representa el primer informe en el Perú de tal asociación. A pesar que los pacientes compartían factores de riesgo vascular, el accidente cerebrovascular isquémico pudo ser una manifestación asociada a la infección por SARSCoV-2 severa, desencadenada por el estado de hiperinflamación e hipercoagulabilidad sanguínea que este virus produce. Asimismo, a pesar que nuestros casos no son generalizables, recomendamos actuar con cautela en el momento de diagnosticar y estudiar un paciente con $\mathrm{ACV}$ en el contexto de la pandemia, pues podría corresponder a una manifestación asociada del COVID-19; por lo que creemos que la TC cerebral diagnóstica del ACV agudo debería extenderse a TC de tórax en busca de un diagnóstico temprano de COVID-19, permitiendo adoptar medidas pertinentes en el manejo inmediato de estos pacientes y en la bioseguridad del personal sanitario.

\section{Correspondencia}

Evelyn Mariños Sánchez

Hospital Nacional Edgardo Rebagliati Martins

Av. Rebagliati 490 Jesús Maria

Teléfono: 511-265-4901 anexo: 3081

Correo electrónico: evelynmarinos1801@gmail.com

Este reporte de casos cuenta con autorización del Comité de ética de IETSI de EsSalud.

Los autores no han recibido financiamiento de ninguna institución o persona. Los autores no tienen conflictos de interés.

\section{REFERENCIAS BIBLIOGRÁFICAS}

1. Huang C, Wang Y, Li X, Ren L, Zhao J, Hu Y, et al. Clinical features of patients infected with 2019 novel coronavirus in Wuhan, China. Lancet. 2020; 395 (10223): 497-506.

2. Lu R, Zhao X, Li J, Niu P, Yang B, Wu H, et al. Genomic characterisation and epidemiology of 2019 novel coronavirus: implications for virus origins and receptor binding. Lancet. 2020; 395: 565-74.

3. Tsai LK, Hsieh ST, Chang YC. Neurological manifestations in severe acute respiratory síndrome. Acta Neurol Taiwan. 2005;14(3):113-9.

4. Kim JE, Heo JH, Kim HQ. Neurological complications during treatment of middle east respiratory síndrome. J Clin Neurol. 2017; 13(3):227-233.

5. Baig A, Khaleeq A, Ali U, Syeda H. Evidence of the COVID-19 Virus Targeting the CNS: Tissue 
distribution, host-virus interaction, and proposed neurotropic mechanisms. ACS Chem Neurosci. 2020; 11:995-8.

6. Moriguchi T, Harii N, Goto J, Harada D, Sugawara $\mathrm{H}$, Takamino J. A first case of meningitis/encephalitis associated with SARS-Coronavirus-2. Int J Infect Dis. 2020; 94:55-58. doi: 10.1016/j.ijid.2020.03.062

7. Toscano G, Palmerini F, Ravaglia S, Ruis L, Invemizzi P, Cuzzoni MG, et al. Guillain -Barré Syndrome Associated with SARS-CoV-2. N Engl J Med. 2020; por publicarse. Doi: 10.10.56/NEJMc2009191

8. Grau AJ, Buggle F, Becher H, Zimmermann E, Spiel $\mathrm{M}$, Fent $\mathrm{T}$, et al. Recent bacterial and viral infection is a risk factor for cerebrovascular ischemia: clinical and biochemical studies. Neurology. 1998; 50(1): 196-203.

9. García-Moncó J, Erro M, García D, Ezpeleta D. Cuadros clínicos neurológicos asociados a la infección por SARS-CoV-2. En: Ezpeleta D, editor. Manual COVID-19 para el neurólogo general. Madrid: Ediciones SEN; 2020. p. 36-46.

10. Mao L, Jin H, Wang M, Hu Y, Chen S, He Q, et al. Neurologic Manifestations of Hospitalized Patients With Coronavirus Disease 2019 in Wuhan, China. JAMA Neurol 2020; por publicarse. Doi:10.1001/ jamaneurol.2020.1127

11. Li Y, Wang M, Zhou Y, Chang J, Xian Y, Mao L, et al. AcuteCerebrovascularDisease Following COVID-19: A Single Center, Retrospective, Observational Study. Lancet. 2020; por publicarse. http://dx.doi. org $/ 10.2139 /$ ssrn. 3550025

12. Ministerio de Salud. MINSA procesó 155 muestras por coronavirus covid-19 y uno re4sultó positivo. Lima: Mnisterio de Salud; 2020. Disponible en: https://www.gob.pe/institucion/minsa/ noticias/108937-minsa-proceso-155-muestraspor-coronavirus-covid-19-y-una-resulto-positivo. $30 / 05 / 2020$

13. Escobar G, Matta J, Taype W, Ayala R, Amado J. Características clínico epidemiológicas de Pacientes fallecidos por COVID-19 en un hospital nacional de Lima, Perú. Rev Fac Med Hum. 2020; 20(2):14-19.

14. Guzmán-Del Giudice O, Lucchesi-Vásquez E, Trelles-De Belaúnde M, Pinedo-Gonzales R, CamereTorrealva M, Daly A, et al. Características clínicas y epidemiológicas de 25 casos de COVID-19 atendidos en la Clínica Delgado de Lima. Rev Soc Peru Med Interna. 2020; 33(1):15-24.

15. Umapathi T, Kor AC, Venketasubramanian N, Chuan $\mathrm{B}$, Tsai $\mathrm{T}$, Chuan $\mathrm{C}$, et al. Large artery ischaemic stroke in severe acute respiratory syndrome (SARS). J Neurol. 2004; 251(10): 1227-31.
16. Wu Z, McGoogan JM. Characteristics of and Important Lessons From the Coronavirus Disease 2019 (COVID-19) Outbreak in China: Summary of a report of 72314 cases from the Chinese center for disease control and prevention. JAMA. 2020; 323(13): 1239-42.

17. World Health Organization. Clinical management of severe acute respiratory infection when novel coronavirus $(\mathrm{nCoV})$ infection is suspected. Ginebra: World Health Organization; January, 2020. Disponible en: https://www.who.int/publications-detail/clinicalmanagementof-severe-acute-respiratory-infectionwhen-novel-coronavirus-(ncov)-infection-issuspected. 28/02/2020.

18. Aggarwal G, Lippi Giuseppe, Michael B. Cerebrovascular disease is associated with an increased disease severity in patients with coronavirus disease 2019 (COVID-19): A pooled analysis of published literatura. INT J STROKE. 2020; Por publicarse. Doi: 10.1177/1747493020921664

19. Helms J, Kremer S, Merdji H, Clere-Jehl, Schenck $\mathrm{M}$, Kummerlen $\mathrm{C}$, et al. Neurological features in Severe SARS- CoV-2 infection. N Engl J Med. 2020; por publicarse. Doi: 10.1056/NEJMc2008597

20. Klok F, Kruip M, Van-der-Meer N, Arbous M, Gommers D, Kant K, et al. Incidence of thrombotic complications in critically ill ICU patients with COVID-19. Thromb Res. 2020; por publicarse. Doi: org/10.1016/j.thromres.2020.04.013

21. Iadecola C, Anrather J. The immunology of stroke: from mechanisms to translation. Nat Med. 2011; 17(7): 796-808.

22. Wang D, Hu B, Hu C, Zhu F, Liu X, Zhang J, et al. Clinical Characteristics of 138 Hospitalized Patients With 2019 Novel Coronavirus-Infected Pneumonia in Wuhan, China. JAMA. 2020; por publicarse. Doi: 10.1001/jama.2020.1585

23. Zhang J, Dong X, Cao Y, Yuan Y, Yang Y, et al. Clinical characteristics of 140 patients infected with SARS-CoV-2 in Wuhan, China. Allergy. 2020; por publicarse. Doi: 10.1111/all.14238

24. Siddiqu HK, Mehra MR. COVID-19 Illness in Native and Immunosuppressed States: A clinical therapeutic staging proposal. J Heart Lung Transplant. 2020; 39(5): 405-407. doi: 10.1016/j.healun.2020.03.012

25. Sociedad española de Enfermedades Infecciosas y Microbiología Clínica. Instituto de Salud Carlos III- Gobierno de España. Interpretación de las pruebas diagnósticas frente a SARS-CoV-2.Madrid: Sociedad españoladeEnfermedades Infecciosasy Microbiología Clínica; 2020. Disponible en: https://www.mscbs. gob.es/profesionales/saludPublica/ccayes/ alertas Actual/nCov-China/documentos/ INTERPRETACION_DE_LAS_PRUEBAS.pdf 28/04/2020. 